\title{
Consideraciones sobre la repasivación de las estructuras corroídas de hormigón $\operatorname{armado}^{(\cdot)}$
}

\author{
Alfonso Cobo*, María Nieves González*, Eduardo Otero* y José Antonio González**
}

Resumen Se analizan las respuestas de electrodos de acero limpios y precorroídos en soluciones saturadas de $\mathrm{Ca}(\mathrm{OH})_{2}$ y en mortero de cemento, recurriendo para ello a técnicas gravimétricas, metalográficas y electroquímicas, esencialmente a medidas de resistencia de polarización. El trabajo trata de encontrar respuestas a algunas cuestiones importantes relativas a la corrosión de las estructuras de hormigón armado (EHA) que, a pesar de parecer elementales, siguen planteando controversias en las vertientes científica, técnica y económica, como las siguientes: ¿Qué velocidades de corrosión son peligrosas en las EHA? ¿Qué resistividades del hormigón garantizan durabilidades suficientes a las EHA? ¿Es posible detener una corrosión ya iniciada? ¿Se pueden repasivar las EHA ya corroídas? ¿Cuándo son eficaces los métodos electroquímicos de rehabilitación de las EHA? Los resultados obtenidos indican que la extracción electroquímica de cloruros y la realcalinización electroquímica no son capaces de repasivar las armaduras fuertemente corroídas. Sin embargo, se pueden considerar métodos eficaces de prevención, si se recurre a ellos antes de que se desencadene la corrosión en estado activo del acero de refuerzo. Si se aplican demasiado tarde resultan ineficaces.

Palabras clave Acero. Hormigón. Estructuras precorroídas. Rehabilitación electroquímica. Repasivación.

\section{Considerations on the repassivation of corroded reinforced concrete structures}

\begin{abstract}
An analysis is made of the responses of clean and precorroded steel electrodes in $\mathrm{Ca}(\mathrm{OH})_{2}$ saturated solutions and in cement mortar, using gravimetric, metallographic and electrochemical techniques, essentially polarisation resistance measurements. The paper aims to answer some important questions about the corrosion of reinforced concrete structures (RCS) which, though seemingly elementary, continue to arouse controversy in scientific, technical and economic circles, such as the following: What corrosion rates are dangerous in RCS? What concrete resistivities guarantee sufficient durability of RCS? Is it possible to detain corrosion once it has begun? Can corroded RCS be repassivated? Are electrochemical RCS rehabilitation methods efficient, and if so, when? The results obtained indicate that electrochemical chloride removal and realkalisation cannot repassivate heavily corroded steel surfaces, however they can be effective methods to prevent corrosion provided they are used before the transition from the passive state to the active one occurs. If applied to late, are useless for this purpose.
\end{abstract}

Keywords Steel. Concrete. Prerusted structures. Electrochemical rehabilitation. Repassivation.

\section{INTRODUCCIÓN}

\subsection{Consideraciones generales}

Se considera tradicionalmente que los refuerzos de acero del hormigón armado (HA) se encuentran en el estado pasivo, protegidos contra la corrosión ${ }^{[1}$ y 2$]$. Esta protección, conferida por una capa pasivante que se forma en la interfase acero/hormigón, autorregenerable, de muy pequeño espesor, en torno a $10 \mathrm{~nm}^{[3]}$, es esencialmente de naturaleza electroquímica, garantizada por la elevada

(•) Trabajo recibido el día 6 de abril de 2000 y aceptado en su forma final el 7 de junio de 2000.

${ }^{*}$ ) Escuela Universitaria de Arquitectura Técnica de Madrid. Avda. Juan de Herrera, 7.28040 Madrid (España).

${ }^{* *}$ Centro Nacional de Investigaciones Metalúrgicas, CENIM (CSIC). Avda. Gregorio del Amo, 8. 28040 Madrid (España). 
alcalinidad del hormigón, usualmente en la región de $\mathrm{pH} 13-14$, y por la existencia de un potencial electroquímico apropiado ${ }^{[4]}$.

Como consecuencia, mientras la corrosión del acero sin proteger representa generalmente un serio problema de mantenimiento en todos los ambientes húmedos, la corrosión del acero embebido en el hormigón queda limitada a unos cuantos casos especiales, que se presentan siempre ligados a la pérdida del estado pasivo, ya sea localmente o en grandes áreas ${ }^{[5}$ y 6$]$.

En ausencia de graves errores en el proceso de fabricación, la pérdida de la pasividad natural de las armaduras del hormigón se debe, la inmensa mayoría de las veces, a los factores desencadenantes siguientes:

- La presencia de iones despasivantes, esencialmente cloruros, en cantidad suficiente para destruir localmente las capas pasivantes.

- $\mathrm{Al}$ descenso del $\mathrm{pH}$ del hormigón, por efecto del $\mathrm{CO}_{2}$ atmosférico, por debajo de un valor crítico, no bien definido, que algunos autores situan en $9,5^{[5]}$.

A pesar de que la corrosión en las EHA sea la excepción y no la regla, con el transcurso del tiempo, mucho más rápidamente en unos ambientes que en otros, se convierte en un problema de proporciones considerables. Como confirmación de esta importancia, baste considerar que, aproximadamente, la mitad de más de los 500.000 puentes de la red de comunicaciones de EE.UU. necesitan reparación, estimándose el coste de su rehabilitación en $2 \times 10^{10} \$$, que se incrementa a razón de $5 \times 10^{8} \$ /$ año $^{[7}$ y 8$]$.

La situación dista mucho de resultar satisfactoria. Contribuye a ello el hecho de que, a pesar de las enormes cantidades de trabajo y de dinero dedicadas al estudio de la corrosión del acero en el hormigón y a la búsqueda de soluciones adecuadas, permanezcan aún como objeto de controversia cuestiones muy importantes, a veces elementales para adoptar decisiones. En este trabajo se pretende plantear algunas de estas cuestiones y proponer respuestas adecuadas. Concretamente, las siguientes:

- La posibilidad de repasivar EHA ya corroídas.

- La existencia (o ausencia) de una relación entre el grado de precorrosión de las armaduras y la posibilidad de repasivación de las mismas.

- Si se puede parar un proceso de corrosión en marcha sin necesidad de repasivar las armaduras.
- Cuándo deben aplicarse los métodos electroquímicos de rehabilitación de las EHA para que resulten eficaces.

\subsection{Dos cuestiones previas sobre el estado actual}

Antes de exponer los ensayos realizados y los resultados obtenidos, permítasenos unas consideraciones previas, como punto de partida para facilitar al lector la evaluación de los mismos. Interesa recordar someramente dos aspectos relacionados con la corrosión de las EHA:

- La cuantía de la corrosión necesaria para producir daños significativos en las EHA.

- Las velocidades de corrosión habituales en tales estructuras, en las circunstancias excepcionales que dan lugar a la pérdida del estado pasivo de los refuerzos.

Respecto al primero de ellos, interesa tener en cuenta que $i_{\text {corr }}=1 \mu \mathrm{A} / \mathrm{cm}^{2}$, aparentemente insignificantes, que sólo suponen una penetración media de la corrosión de 11,6 $\mu \mathrm{m} /$ año en el caso del acero, resultan muy peligrosas en las estructuras de hormigón armado, en las que pueden provocar grietas en el recubrimiento de hormigón para penetraciones de la corrosión inferiores a $100 \mu \mathrm{m}$ (Fig. 1) ${ }^{[9]}$. Agrietamientos a partir de penetraciones de $20 \mu \mathrm{m}$ son mencionados también por otros investigadores ${ }^{[10]}$.

En cuanto al segundo aspecto, la figura 2 pone de manifiesto que la concurrencia de contaminaciones en cloruros por encima de un determinado umbral, que se suele fijar en un $0,4 \%$ en relación al peso de cemento ${ }^{[11]}$ y de una humedad relativa elevada o condiciones de inmersión total o parcial, da lugar a $i_{\text {corr }}$ comprendidas entre 1 y $10 \mu \mathrm{A} / \mathrm{cm}^{2}$ ${ }^{[12]}$, con las que, a la vista de la figura 1 , se pueden temer deterioros significativos en periodos de tiempo inferiores a 10 años.

\section{MATERIALES Y MÉTODOS}

\subsection{Materiales}

Se ha estudiado el comportamiento del acero en soluciones saturadas de $\mathrm{Ca}(\mathrm{OH})_{2}$, empleadas desde hace casi medio siglo para simular la fase acuosa del hormigón armado ${ }^{[13]}$, utilizando como electrodos láminas de acero suave de $40 \times 50 \times 0,5 \mathrm{~mm}$, con una prolongación de $5 \mathrm{~mm}$ de ancho para 


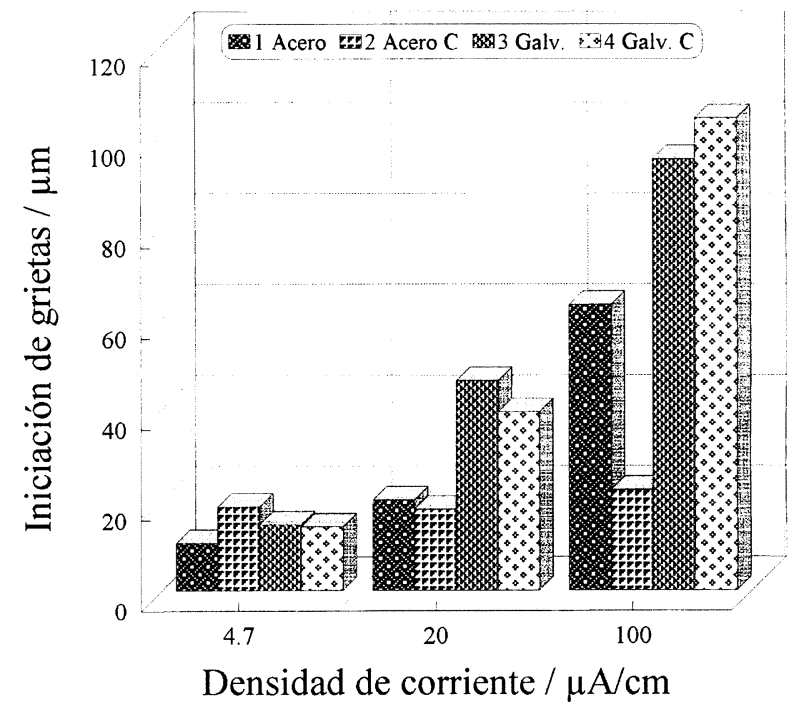

Figura 1. Penetraciones de la corrosión a las que se detectaron iniciación de grietas en el recubrimiento de mortero. Ensayos con pequeñas probetas de $8 \times 5,5 \times 2 \mathrm{~cm}$, carbonatadas y $\sin$ carbonatar, con electrodos de acero para armar y de acero galvanizado ${ }^{[9]}$.

Figure 1. Corrosion penetrations at which the initiation of cracks in the mortar coating is detected. Tests with small specimens of $8 \times 5,5 \times 2 \mathrm{~cm}$, carbonated and uncarbonated, with reinforcement steel and galvanised steel electrodes $^{[9]}$

facilitar el contacto eléctrico. La mencionada prolongación y una cara de las probetas se aislaba con cinta adhesiva protectora quedando una superficie

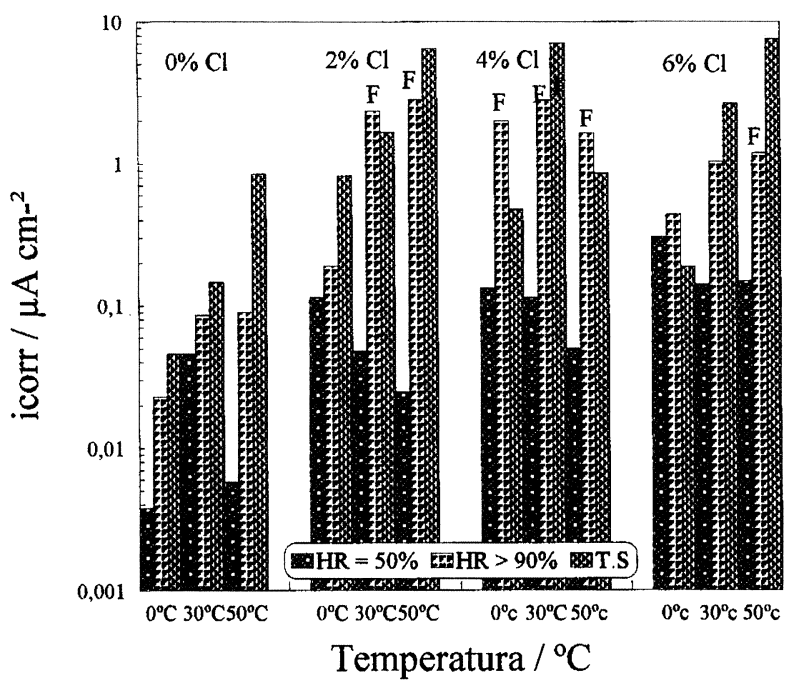

Figura 2. Velocidades de corrosión $\left(i_{\text {corr }}\right)$, determinadas después de 1 año de fabricadas las probetas de mortero, para diferentes adiciones de cloruros y condiciones de humedad relativa y temperatura ${ }^{[12]} . \mathrm{F}=$ probetas con fisuras.

Figure 2. Corrosion rates $\left(i_{\text {corr }}\right)$ determined 1 year after the manufacturing of mortar specimens, for different chloride additions and relative humidity and temperature conditions $^{[12]} . F=$ fissured specimens. activa de $20 \mathrm{~cm}^{2}$, que actuaba de electrodo de trabajo durante las medidas electroquímicas. Como electrodo de referencia se empleó siempre el de calomelanos saturado, empleando un alambre de acero inoxidable como contraelectrodo.

Las soluciones saturadas de $\mathrm{Ca}(\mathrm{OH})_{2}$ se han utilizado para imponer un $\mathrm{pH}$ muy alcalino, con el fin de garantizar la pasivación de los electrodos de acero limpio y provocar, si fuera posible, la repasivación de los corroídos. Por dicha razón se han empleado sin adiciones de cloruros, salvo que expresamente se diga lo contrario.

No obstante, la mayoría de los resultados se han obtenido con pequeñas probetas de mortero de $2 \times 5,5 \times 8 \mathrm{~cm}$, como la de la figura 3 , con dos redondos de acero para armar, de $6 \mathrm{~mm}$ de diámetro, embebidos en posiciones simétricas y un electrodo central, de acero inoxidable, que servía como contraelectrodo, siendo el electrodo de calomelanos saturado la referencia utilizada, como

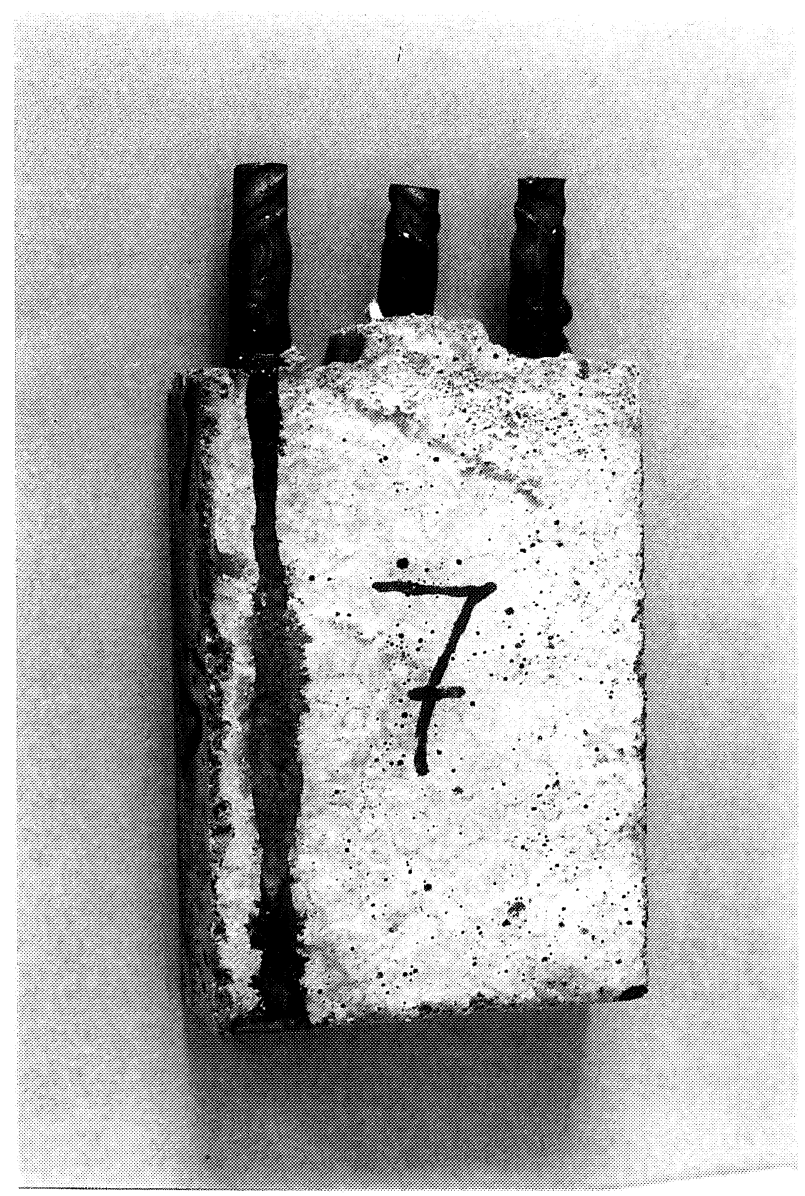

Figura 3. Aspecto de una de las probetas de mortero utilizadas en los ensayos al término de los mismos, mostrando síntomas de corrosión.

Figure 3. Aspect of one of the tested mortar specimens at the end of testing, showing symptoms of corrosion.

Rev. Metal. Madrid 36 (2000) 
en los ensayos realizados en soluciones de $\mathrm{Ca}(\mathrm{OH})_{2}$. Con este tipo de probetas se puede determinar el efecto de los factores de corrosión dependientes del propio material de construcción, de la armadura y del medio ambiente. Sobre los redondos de acero, se delimitaba, con resina epoxi en este caso, una superficie de trabajo de $10 \mathrm{~cm}^{2}$.

Las probetas de mortero se fabricaron con una dosificación de cemento/arena/agua $=1 / 3 / 0,5$, exentas de cloruros en unos casos y, en otros, con la adición de $\mathrm{CaCl}_{2}$ en la proporción de un $3 \%$ en relación al peso de cemento.

\subsection{Técnicas utilizadas}

La evolución con el tiempo del comportamiento de los electrodos se siguió a través de la medida de los potenciales de corrosión $\left(E_{\text {corr }}\right)$ y de la resistencia de polarización $\left(R_{\mathrm{p}}\right)$ para determinar las velocidades de corrosión a partir de la ecuación de Stern y Geary:

$$
i_{\text {corr }}=B \Delta V / S \Delta I=B / R_{\mathrm{p}}
$$

donde $i_{\text {corr }}$ es la intensidad de corrosión, relacionada con la disolución metálica a través de la ley de Faraday; B la constante de la ecuación de Stern, habiendo utilizado en los cálculos un valor intermedio de 0,026 V; S la superficie del electrodo de trabajo y $R_{\mathrm{p}}$ la resistencia de polarización o cociente entre la señal de potencial aplicada al sistema $(\Delta \mathrm{V})$ y la respuesta en corriente del mismo $(\Delta I)$ por unidad de superficie. La $R_{\mathrm{p}}$ se determinó aplicando una señal de $10 \mathrm{mV}$ y midiendo la respuesta en corriente a los $15 \mathrm{~s}$.

\subsection{Condiciones de operación}

Para comprobar el efecto del grado de precorrosión del acero en la posibilidad de repasivación se utilizaron los siguientes tipos de electrodos:

- Probetas planas de $40 \times 50 \mathrm{~mm}$ fabricadas con la chapa de acero suave y trozos de $10 \mathrm{~cm}$ de longitud cortados del redondo de acero para armar de $6 \mathrm{~mm}$ de diámetro, con la superficie limpia en ambos casos, recién decapada en una solución de $\mathrm{HCl}$ al $50 \%$, inhibida con $4 \mathrm{~g} / \mathrm{L}$ de urotropina, lavada a fondo después en agua corriente y agua destilada y secada, inmediatamente, con aire a presión.

- Láminas de acero contaminadas con 500 mg de $\mathrm{Cl}^{-} / \mathrm{m}^{2}$ y expuestas distintos tiempos en cámara húmeda para lograr diferentes grados de preco- rrosión antes de tratar de repasivarlas en la solución saturada de $\mathrm{Ca}(\mathrm{OH})_{2}$.

- Redondos de acero para armar con grados de precorrosión diferentes, que se embebían luego en mortero sin adiciones de cloruros, para facilitar al máximo su repasivación. Los distintos grados de precorrosión se conseguían embebiendo previamente los electrodos en mortero contaminado con cloruros, e imponiendo una densidad anódica de corriente de $10 \mu \mathrm{A} / \mathrm{cm}^{2}$ durante periodos prefijados de tiempo. Una vez preocorroídos los electrodos se rompían las probetas de mortero y se fabricaban otras iguales sin adiciones de cloruros, lo que equivale a una extracción del $100 \%$, inalcanzable en la práctica. Estas probetas se mantenían en una atmósfera de muy elevada humedad relativa, cercana a la saturación, que provoca, de acuerdo con la experiencia, $i_{\text {corr }}$ elevadas, entre 1 y $10 \mu \mathrm{A} / \mathrm{cm}^{2}$ en los electrodos activos y unas 100 veces menores en los pasivos o repasivados.

- Alambres de acero procedentes de elementos estructurales prefabricados de las ventanas de la nave de Metalurgia Extractiva no Férrea del CENIM que, a causa de la corrosión, provocada por la carbonatación del mortero, se agrietaron y desprendieron en el verano de 1998, a los 30 años, aproximadamente, de su fabricación.

\section{RESULTADOS EXPERIMENTALES}

La figura 4, que corresponde a la determinación de un potencial de ruptura en una solución saturada de hidróxido cálcico, contaminada con cloruros, permite realizar una visualización comparativa de los riesgos asociados a los estados activo y pasivo. La curva en línea continua corresponde al estado pasivo, con densidades de corriente muy pequeñas aun para polarizaciones de centenares de $\mathrm{mV}$. En cambio, la curva de retorno, de puntos en la figura, refleja el comportamiento después de la rotura local de la capa pasivante. Puede comprobarse que para los mismos potenciales se obtienen densidades de corriente unas 100-1.000 veces mayores que las correspondientes al estado pasivo. En proporción parecida debería reducirse la durabilidad de las EHA si se corroen en estado activo.

En la figura 5 sólo se pretende recordar, con datos nuevos, la interdependencia de la velocidad de corrosión con la resistividad del hormigón en las estructuras que han perdido la pasividad, hecho ya

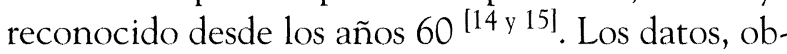
tenidos para distintos grados de saturación de la 


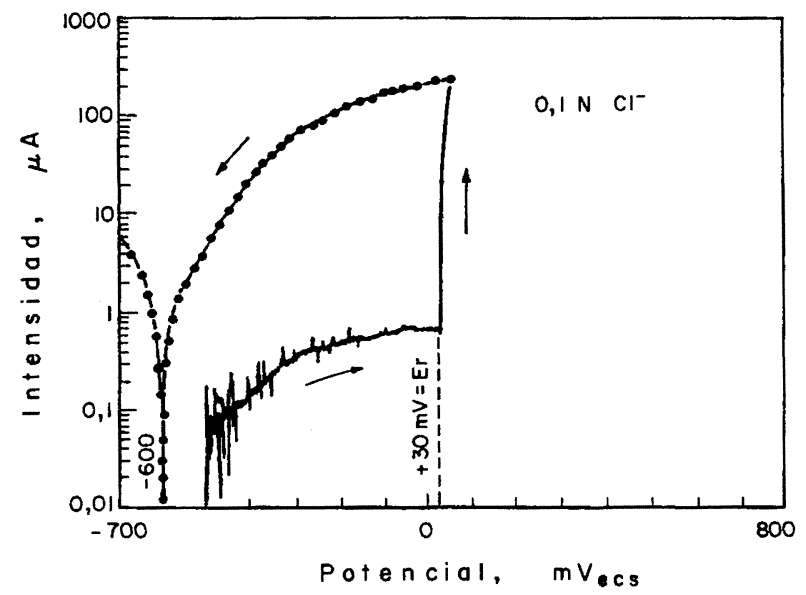

Figura 4. Curva de polarización de acero en solución saturada de $\mathrm{Ca}(\mathrm{OH})_{2}$ con adición de cloruros, mostrando el potencial de ruptura y respuestas en corriente al menos 100 veces mayores después de provocada la transición pasividad-actividad (curva de retorno). Velocidad de polarización: $1 \mathrm{mV} / \mathrm{s}$.

Figure 4. Polarisation curve of steel in $\mathrm{Ca}(\mathrm{OH})_{2}$ saturated solution with chloride additions, showing breakdown potential and current responses at least 100 times greater after provocation of the passive-active transition (return curve). Potential sweep rate: $1 \mathrm{mV} / \mathrm{s}$.

red de poros del mortero, se refieren a una probeta con adición de un $3 \%$ de $\mathrm{CaCl}_{2}$ y, como contraste, otra sin aditivos despasivantes. Existe, en coordenadas logarítmicas, una proporcionalidad inversa entre la resistividad del hormigón y la velocidad de corrosión de las armaduras, con un campo amplísimo de variabilidad.

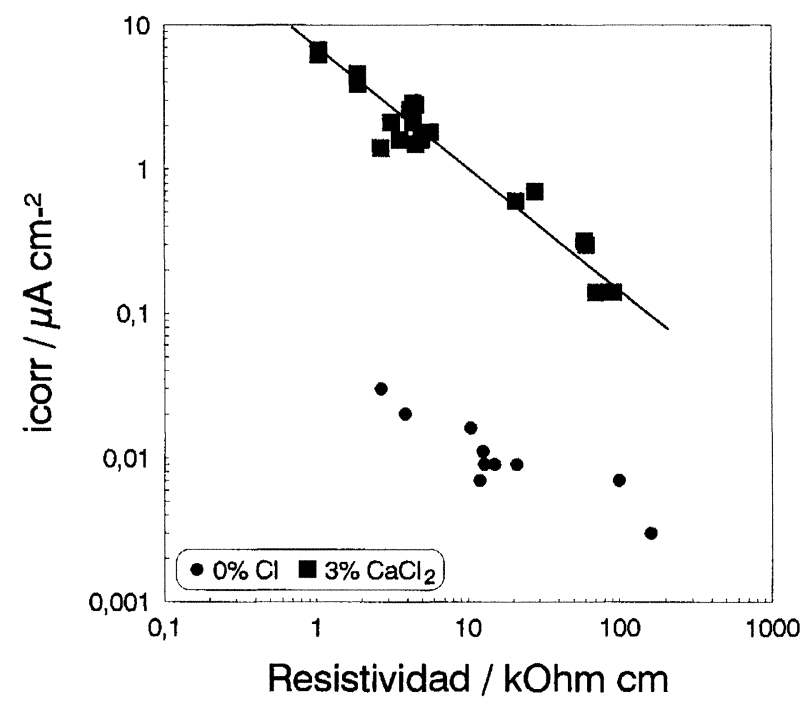

Figura 5. Relación entre las $i_{\text {corr }}$ y la resistividad de morteros con y sin adiciones de cloruros, al cambiar el grado de saturación de la red de poros.

Figure 5. Relationship between the $\mathrm{i}_{\text {corr }}$ and resistivity of mortars with and without chloride additions, as the degree of pore network saturation changes.
Los resultados de la figura 6 , obtenidos en soluciones saturadas de $\mathrm{Ca}(\mathrm{OH})_{2}$ exentas de cloruros, ofrecen pruebas convincentes de la gran importancia que el grado de precorrosión de los electrodos ejerce sobre la repasivación de los mismos. Las superficies de acero limpias se pasivan de forma rápida, por efecto del elevado $\mathrm{pH}$ de la solución. Las superficies precorroídas muestran, en cambio, después de un mes de ensayo, velocidades de corrosión mucho más elevadas. Es más, las $i_{\text {corr }}$ a las que se tiende son tanto mayores cuanto más alto es el grado de precorrosión inicial. Efectivamente, las curvas a), b), c) y d) de la figura, corresponden a precorrosiones de 0, 145, 280 y $4.300 \mathrm{mg} \mathrm{Fe} / \mathrm{dm}^{2}$, respectivamente. Los potenciales de corrosión no ofrecen, en cambio, información importante.

Las macrografías b), c) y d) de la figura 7 permiten visualizar los grados de precorrosión de los electrodos a los que corresponden las curvas de la figura 6 con idéntica designación. No se incluye macrografía para el caso de la curva a), con una superficie totalmente limpia (recién decapada). En el extremo opuesto, puede comprobarse, para el electrodo de la curva d), cortado de los alambres de refuerzo de la ventana de la nave de Metalurgia Extractiva no Férrea del CENIM, una superficie totalmente cubierta por productos de corrosión.

Lo expuesto para las soluciones saturadas de $\mathrm{Ca}(\mathrm{OH})_{2}$ resulta aplicable para los redondos corrugados precorroídos embebidos en morteros de

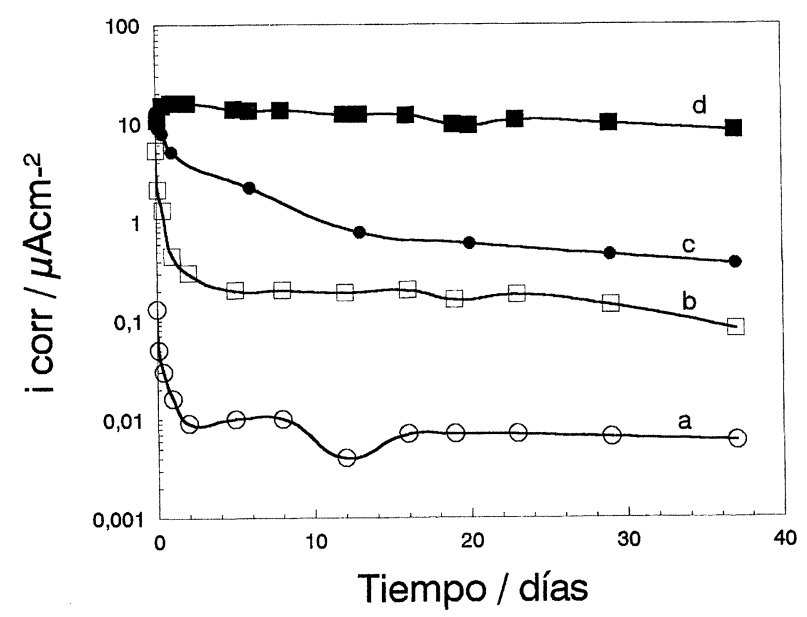

Figura 6. Evolución de las $i_{\text {corr }}$ con el tiempo, en solución saturada de $\mathrm{Ca}(\mathrm{OH})_{2}$ sin adiciones de cloruros, para distintos grados de precorrosión del acero: a) 0, b) 145, c) 280 y d) $4.300 \mathrm{mg} / \mathrm{dm}^{2}$ de hierro corroído.

Figure 6. Evolution of $\mathrm{i}_{\text {corr }}$ with time, in $\mathrm{Ca}(\mathrm{OH})_{2}$ saturated solution without chloride additions, for different degrees of steel precorrosion: a) 0, b) 145 , c) 280 and d) 4,300 $\mathrm{mg} / \mathrm{dm}^{2}$ of corroded iron. 

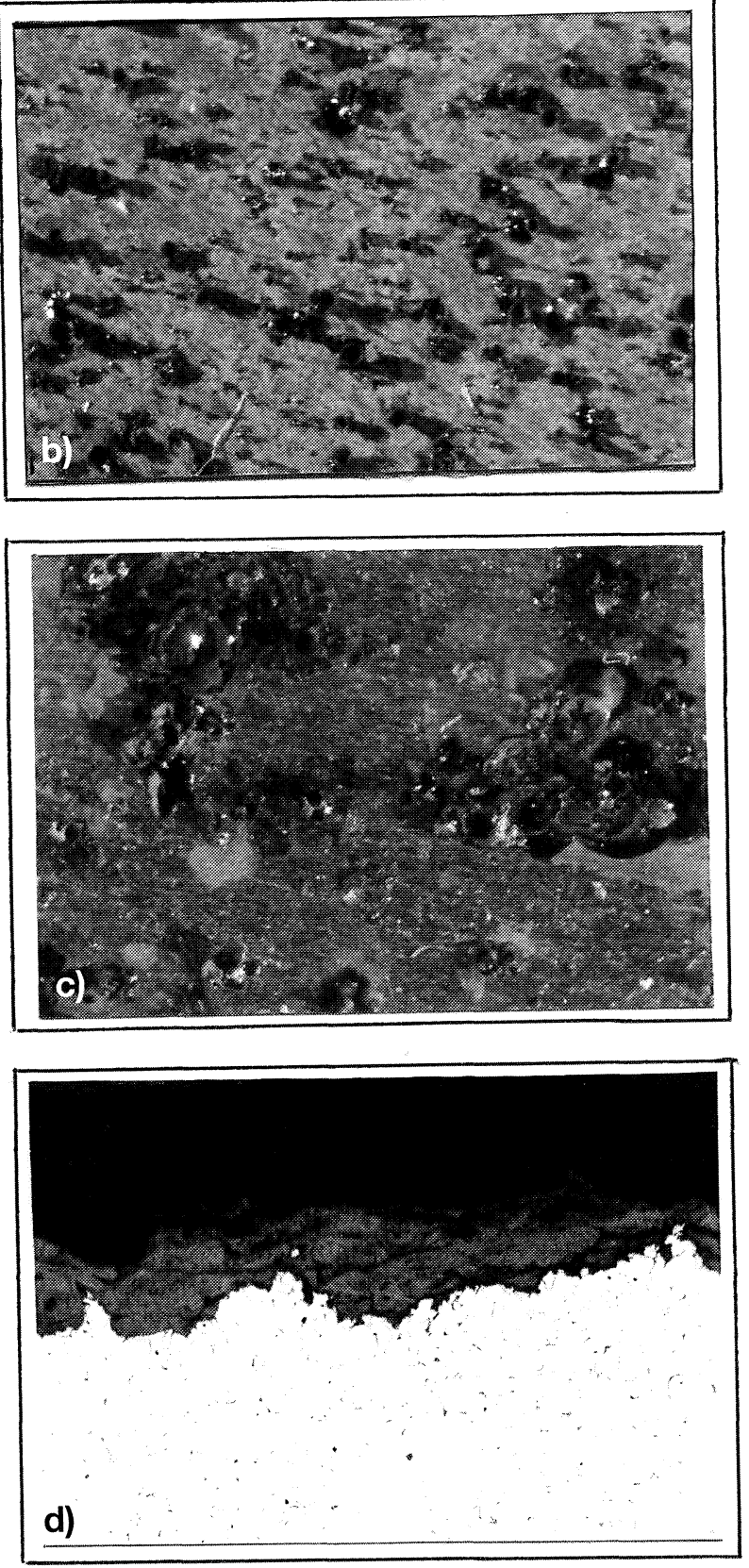

Figura 7. Aspecto de la superficie de los electrodos a los que corresponden las curvas de la figura anterior: b) y c) macrografías: $x 10 ;$ d) micrografía: x100.

Figure 7. Surface aspect of the electrodes corresponding to the curves in the previous figure: b) and c) macrographs at 10 magnifications and d) micrograph at 100 magnifications.

cemento totalmente exentos de cloruros, como demuestran, sin lugar a dudas, los resultados de la figura 8 , en la que se relacionan las $i_{\text {corr }}$ obtenidas al mes de fabricadas las probetas, con el grado de precorrosión, determinado al final de los ensayos por disolución de los productos de corrosión y pesada. La corrosión producida durante el ensayo sólo representa un $10 \%$, como máximo, de la pérdi-

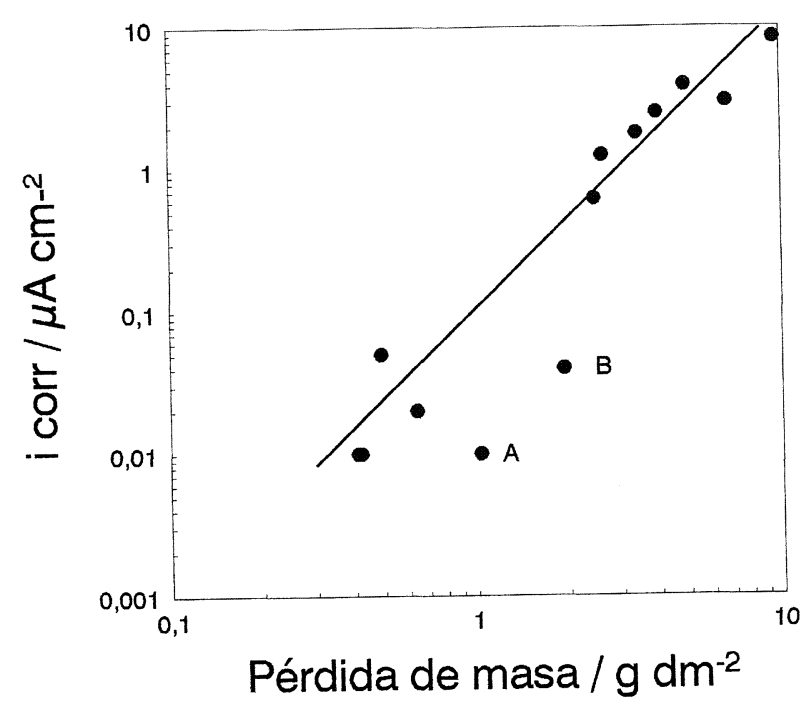

Figura 8. Relación entre el grado de precorrosión de los electrodos y sus $i_{\text {corr }}$ al mes de embebidos en probetas de mortero exentas de cloruro.

Figure 8. Relationship between the degree of electrode precorrosion and their $\mathrm{i}_{\text {corr }}$ one month after embedding in chloride free mortar specimens.

da de masa total, que se debe, casi exclusivamente, a la precorrosión provocada de los electrodos. No contradicen esta apreciación los puntos A y B, con una respuesta mucho mejor de la previsible, porque la precorrosión en tales electrodos tuvo lugar bajo la pintura epoxi, que delimitaba la superficie libremente expuesta al medio, y dicha zona no quedó luego sometida a la acción del mortero sin cloruros, razón por la cual se han obtenido respuestas típicas de electrodos pasivos.

\section{DISCUSIÓN}

\section{1. ¿Qué velocidades de corrosión son peli- grosas en las EHA?}

A partir de los primeros años del siglo XX se difundió el uso de las EHA de un modo progresivo, $y$ parece que debería haberse establecido ya un pleno acuerdo sobre las velocidades de corrosión de los refuerzos que plantean graves problemas de durabilidad. Sorprendentemente, persisten opiniones muy diferentes al respecto ${ }^{[5}$ y 16$]$. En la figura 9 se ofrece el punto de vista de Clear ${ }^{[7}$ y 17] , que los autores juzgan entre los más acordes con la realidad.

De la observación de las figuras 1 y 2 , a las que ya se hizo referencia en la Introducción se infiere: que $i_{\text {corr }} \geq 1 \mu \mathrm{A} / \mathrm{cm}^{2}$ deben considerarse de elevado riesgo en las EHA, lo que concuerda, muy aproximadamente, con las orientaciones de Clear (Fig. 9). 


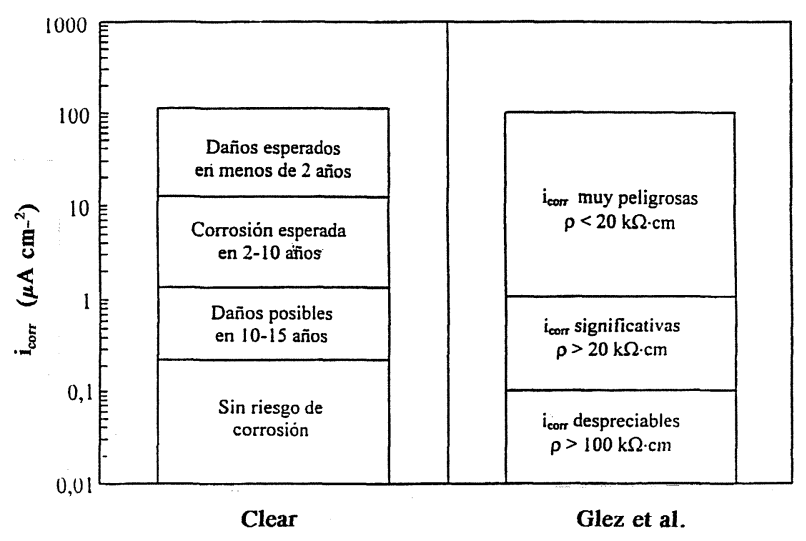

Figura 9. Riesgos asociados a distintos niveles de velocidades de corrosión, según Clear ${ }^{[17]}$ y González et al. ${ }^{[15]}$.

Figure 9. Risks associated with different corrosion rate levels, according to Clear ${ }^{[17]}$ and González et a [15].

Las figuras 4 y 6 informan de una relación de 100 a 1, poco más o menos, entre las velocidades de corrosión de los estados activo y pasivo, de manera que una repasivación eficaz debería suponer reducciones de las $i_{\text {corr }}$ en torno al $99 \%$. No pueden aceptarse como enteramente satisfactorias reducciones del $75 \%$, anotadas a veces en la bibliografía como resultado de la aplicación de inhibidores, con o sin extracción de cloruros ${ }^{[7 \text { y } 18]}$, o velocidades de penetración de 0,3-0,4 mpy (7,6-10,1 $\mu \mathrm{m} / \mathrm{año}$ ), obtenidas meses después de extraídos los cloruros $^{[19]}$, que conducirían al agrietamiento de los recubrimientos de hormigón en pocos años (Fig. 1).

\section{2. ¿Qué resistividades del hormigón garanti- zan durabilidades suficientes a las EHA?}

La cinética de corrosión depende enormemente de la resistividad del hormigón, $\rho$, según ponen de manifiesto los resultados de la figura 5. La posibilidad de corrosión significativa está siempre ligada a la pérdida del estado pasivo, causada por la contaminación en cloruros o la carbonatación del hormigón ${ }^{[1}$ y $^{5]}$. Pero, para que tal posibilidad se transforme en una reducción preocupante de durabilidad, es necesaria la concurrencia de un grado de humedad tal que reduzca la $\rho$ por debajo de $100.000 \Omega \mathrm{cm}$ (Fig. 5), pues para resistividades superiores se obtendrían $i_{\text {corr }} \leq 0,1 \mu \mathrm{A} / \mathrm{cm}^{2}$, similares a las que se producen en las estructuras pasivas.

\section{3. ¿Es posible detener una corrosión ya ini- ciada?}

Enlazando con la cuestión precedente, podría asegurarse que el control de humedad, el método más antiguo de protección de las estructuras metálicas, resultaría también eficaz en las EHA (Fig. 5). La pasividad natural conferida por el cemento a las armaduras evita la necesidad de recurrir a bajos niveles de humedad pero, en el caso de las estructuras activas, eliminar la humedad hasta conseguir $\rho>100.000 \Omega \mathrm{cm}$ y evitar el posterior ingreso de la misma significaría dar una respuesta positiva a la pregunta que inicia este apartado, además sin necesidad de repasivar las armaduras. Otra cosa es que el procedimiento resulte posible y rentable.

Otro método eficaz, capaz de detener un proceso ya iniciado de corrosión, del que no se aportan datos originales, pero que está suficientemente avala-

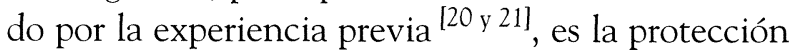
catódica (PC). A pesar de que el escaso espesor de los recubrimientos de hormigón dificulta la distribución uniforme de la corriente de protección, las dificultades técnicas están actualmente resueltas y se ha comprobado que la PC es la única técnica de rehabilitación capaz de detener los procesos de corrosión en marcha en los puentes de hormigón de las regiones frías, tratados con sales de deshielo, cual-

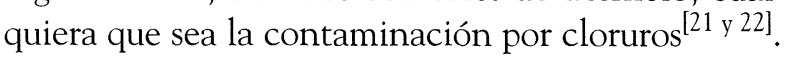

\section{4. ¿Se pueden repasivar las EHA ya corroídas?}

Se trata de la pregunta capital, la inquietud que impulsó la iniciación del trabajo. La realcalinización del hormigón y la extracción electroquímica de cloruros, métodos de rehabilitación de las EHA que suscitan actualmente el máximo interés, nacieron en la esperanza de que, eliminadas las causas de la corrosión (ya sean los cloruros o la carbonatación) se detuviera ésta, al repasivarse las armaduras.

Las figuras 6 y 8 resultan sumamente ilustrativas para enjuiciar la posibilidad de repasivación. Ni la realcalinización más perfecta, ni la extracción más completa de cloruros conseguirían situaciones tan favorables como las representadas por las figuras 6 y 8 , para la repasivación de las estructuras corroídas, una solución saturada de $\mathrm{Ca}(\mathrm{OH})_{2}$ y un mortero de cemento recién fabricado, respectivamente, ambos medios totalmente exentos de cloruros. En efecto, la realcalinización consigue elevar el $\mathrm{pH}$ de la fase acuosa del hormigón hasta los valores propios de las estructuras nuevas, pero no recupera la reserva alcalina del hormigón recién fabricado ${ }^{[23]}$; las fases sólidas carbonatadas continuan como carbonatos. La extracción de cloruros, por otra parte, no consigue nunca reducir su contenido a los niveles encontrados en morteros u hormigones fabricados con materias primas exentas de cloruros. 
Las figuras 6 y 8 parecen desterrar la posibilidad de repasivación. En efecto, las $i_{\text {corr }}$ finales llegan a diferir más de dos órdenes de magnitud entre la superficie limpia (curva "a" de la Fig 6) y la totalmente cubierta de productos de corrosión, como es el caso de los alambres procedentes de las ventanas de la nave de Metalurgia Extractiva del CENIM (curva y micrografía "d" de las Figs. 6 y 7, respectivamente). La consideración conjunta de las figuras 6 y 7 permite afirmar que cuanto mayor es el grado inicial de ataque, mayores son las velocidades de corrosión que se pueden esperar después de eventuales tratamientos de realcalinización y extracción electroquímica de cloruros. Quizá las $i_{\text {corr }}$ finales sean proporcionales al porcentaje de superficie inicialmente corroída.

En el caso de una hipotética extracción total de cloruros del mortero u hormigón (Fig. 8), puede comprobarse que existe también, como en el caso de soluciones saturadas de $\mathrm{Ca}(\mathrm{OH})_{2}$, una evidente proporcionalidad, en escala logarítmica, entre el grado de precorrosión y las $i_{\text {corr }}$ finales. El comportamiento de los puntos A y B, aparentemente anormal, es atribuible a la corrosión sufrida bajo la resina epoxi que delimitaba la superficie expuesta al medio.

Sólo parece factible repasivar acero con grados incipientes de corrosión ${ }^{[24]}$. Las figuras 6,7 y 8 aportan argumentos suficientes, a nuestro entender, para asegurar que no es posible la repasivación de superficies de acero con grados de precorrosión altos $\mathrm{o}$, incluso, moderados.

No deberían extrañar los resultados obtenidos, pues cualquier recomendación sobre pasivación de materiales metálicos pasa por una limpieza previa, escrupulosa, de la superficie de los mismos. Si fuera posible la repasivación de las superficies de acero fuertemente corroídas: ¿Qué sentido tendría exigir, en los procedimientos tradicionales de reparación de las EHA, la limpieza a fondo de todo el perímetro de las armaduras, una vez eliminado el hormigón carbonatado o contaminado con cloruros ${ }^{[25]}$ antes de aplicar el material de reparación? Los resultados avalan las exigencias de los procedimientos tradicionales, pero establecen serias limitaciones, como se analiza a continuación, a la utilización de los procedimientos de rehabilitación electroquímica de las EHA ya corroídas.

\section{5. ¿Cuándo son eficaces los métodos electro- químicos de rehabilitación de las EHA?}

No se está negando el atractivo de la realcalinización y la extracción de cloruros como métodos de rehabilitación electroquímica de las EHA, se está tratando de matizar los riesgos de su utilización indiscriminada. El atractivo de dichos métodos se promociona intensamente en la actualidad: frente a los métodos de reparación tradicionales por resultar mucho menos dañinos para los operarios y mucho más respetuosas con el entorno; y frente a la $\mathrm{PC}$, un

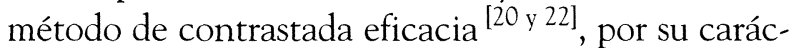
ter temporal, de pocas semanas de duración, a diferencia del carácter permanente de la PC.

La extracción de cloruros y la realcalinización son hechos repetidamente comprobados, que suelen presentarse, erróneamente a juicio de los autores, como éxitos en sí mismos. A nuestro entender, ambos métodos de rehabilitación no pueden considerarse como un fin, sino como un medio para garantizar la durabilidad de las EHA, lo que, si se aplican a estructuras ya dañadas por corrosión, requiere la repasivación de las armaduras.

En cambio, si mediante inspecciones preventivas se recurre a la realcalinización antes de que el frente de carbonatación alcance el nivel de las armaduras, o a la extracción de cloruros antes de que estos iones penetren hasta la superficie del acero en cantidad peligrosa, se podrá prolongar la vida en servicio de las EHA indefinidamente, extendiendo, si es necesario mediante actuaciones repetidas, el periodo de iniciación de la corrosión hasta alcanzar la durabilidad prevista en proyecto.

Sin embargo, raramente se solicitan inspecciones antes de que aparezca sintomatología visible de la corrosión, y esto, en las EHA, dada la inaccesibilidad visual de las armaduras, significa que suele acudirse demasiado tarde, cuando el proceso de corrosión ya ha avanzado y, de acuerdo con los resultados de este trabajo, la repasivación de las armaduras es problemática, si no imposible.

\section{CONCLUSIONES}

- Los resultados obtenidos permiten asegurar que no es posible la repasivación de superficies de acero con grados de precorrosión altos o, incluso, moderados.

- Cuanto mayor es el grado de precorrosión del acero, o el porcentaje de superficie corroída, tanto mayor es su velocidad de corrosión en morteros de cemento exentos de cloruros, o en soluciones que simulen la fase acuosa de la red de poros del hormigón.

- Es de temer, por tanto, que los métodos electroquímicos de rehabilitación de las EHA, bien sea la realcalinización o la extracción de 
cloruros, sean incapaces de repasivar EHA ya corroídas.

- Tanto la realcalinización como la extracción electroquímica de cloruros se pueden considerar como métodos eficaces de prevención, si se recurre a ellos antes de que el frente de carbonatación o los cloruros lleguen al nivel de las armaduras, pero no como métodos de rehabilitación de las EHA ya deterioradas por la corrosión.

- Si se quiere garantizar una durabilidad suficiente a las EHA, incluso en el caso de que los refuerzos se hallen en el estado activo, es preciso reducir el porcentaje de saturación de la red de poros del hormigón hasta obtener resistividades $\geq 100 \mathrm{k} \Omega \mathrm{cm}$, que dan lugar a $i_{\text {corr }} \leq 0,1$ $\mu \mathrm{A} / \mathrm{cm}^{2}$, con penetraciones de la corrosión de $1 \mu \mathrm{m} /$ año, o inferiores. En muchos casos, sin embargo, el procedimiento no es factible.

\section{REFERENCIAS}

[1] C. L. Page y K.W.J. Treadaway: Nature, 297 (1982) 109.

[2] 12-CRC Committee: Mater. Const. 9 (1976) 187.

[3] J.R. Gancedo, C. Alonso, C. Andrade y M. Gracia, Corrosion 45 (1989) 976.

[4] K.K. Sagoe-Crentsil y F.P. Glasser, Mag. Concr. Res. 41 (1989) 205.

[5] J.A. González, S. Feliu, P. Rodríguez, E. Ramirez, C. ALONSO y C. ANDRADE, Mater. Struct. 29 (1996) 40.

[6] J.A. González, S. Feliu, P. Rodríguez, W. López, E. Ramirez, C. Alonso y C. Andrade, Mater. Struct. 29 (1996) 97.

[7] W.D. Collins, R.E. Weyers e I.L. Al-QAad, Corrosion 49 (1993) 74-78.

[8] Strategic Highway Research Program, Participant Workbook-National Workshop: Washington, DC, Sept. 1985, pp. TRA 4-1.
[9] J.A. González, C. Andrade, P. Rodriguez, C. Alonso y S. FELIU, Effect of corrosion current on the degradation of reinforced concrete structures, en Progress in the understanding and prevention of corrosion. Eds. J.M. Costa y A.D. Mercer, The Inst. of materials y la Sociedad Española de Química Industrial. Reino Unido, 1993, pp. 629-633.

[10] C. AndRade, C. Alonso y M. Molina, Mater. Struct. 26 (1993) 453

[11] Instrucción de Hormigón Estructural EHE-98 (Ministerio de Fomento, Madrid).

[12] W. López, J.A. González y C. Andrade, Cem. Concr. Res. 23 (1993) 1130-1140.

[13] H. KAESCHE, Zement-Kalk-Gips 12 (1959) 289.

[14] R.F. StRatfull, Mater. Prot. 7 (1968), 29.

[15] J.A. González, M. Benito, A. Bautista y E. Ramirez, Rev. Metal. Madrid 30 (1994) 271-282.

[16] J.P. BROOMFIELD, Life prediction of corrodible structures, NACE Symposium, Cambridge, Sept. 1991, Paper $n^{\circ}$ 14.

[17] K.C. CleAR Inc. 3LP Package:Tests Procedures. Data Analysis and General Information, July 1990. Appendix, p. 9.

[18] S. Hetiarachi y A.T. Gaynor, Mater. Prot., (3) (1992) 62.

[19] J.E. Slater, D.R. Lankard y P.J. Moreland, Mater. Perf. Nov. (1976) 21.

[20] M.A. Gullín, Rev. Iberoam. Corros. Prot. 17 (1987) 99.

[21] B.S. WYAT y D.J. IRVINE, Mater. Perf. 26 (1987) 12.

[22] R.A. BARNHART, FHWA Memorandum, Federal Highway Administrartion, Abril 1982.

[23] J. MIETZ, Electrochemical rehabilitation methods for reinforced concrete structures. Publication $n^{\circ} 24$ of the European Federation of Corrosion. The Institute of Materials, London (Reino Unido), 1998.

[24] J.A. González, A Cobo, M.N. González y E. Otero, Mater. Corros. 51 (2000) 97.

[25] M. Fernández Cánovas, Patología y terapéutica del hormigón armado, $2^{\mathfrak{a}}$ ed. Ed. Dossat S.A. Barcelona, 1984, pp. 193-222. 